\title{
Modelling the Microstructure and Stress in Dense Suspensions Under Inhomogeneous Flow
}

\author{
J. J. J. Gillissen ${ }^{1, \text { * } \text { and C. Ness }}{ }^{2}$ \\ ${ }^{1}$ Department of Mathematics, University College London, \\ Gower Street, London WC1E 6BT, United Kingdom \\ ${ }^{2}$ School of Engineering, University of Edinburgh, Edinburgh EH9 3FB, United Kingdom
}

(Dated: October 1, 2020)

\begin{abstract}
Under inhomogeneous flow, dense suspensions exhibit complex behaviour that violates the conventional homogenous rheology. Specifically, one finds flowing regions with a macroscopic friction coefficient below the yielding criterion, and volume fraction above the jamming criterion. We demonstrate the underlying physics by incorporating shear rate fluctuations into a recently proposed tensor model for the microstructure and stress, and applying the model to an inhomogeneous flow problem. The model predictions agree qualitatively with particle-based simulations.
\end{abstract}

Introduction.- Many materials, such as foods, cosmetics and ceramic precursors, consist of particles densely suspended in liquid, and their production relies on understanding the corresponding fluid mechanics [1. Despite a century of intense research including recent progress [2], comprehensive theoretical models are still lacking [3]. Indeed, even the simple case of non-Brownian, non-inertial, hard spheres remains desceptively challenging 4. Under simple shear flow the mechanics are, in principle, governed by a single dimensionless parameter: specifying only the macroscopic friction coefficient $\mu=\Sigma_{x y} / \Pi$ sets the remaining nondimensional variables, viz., the volume fraction $\phi$ and the non-dimensional shear rate $S=\eta_{s} \dot{\gamma} / \Pi$ [5]. Here $\Sigma_{x y}$ is the shear component of the stress tensor $\Sigma_{i j}$, $\Pi=-D^{-1} \Sigma_{i i}$ is the pressure (in $D$ dimensions), $\eta_{s}$ is the viscosity of the suspending medium, and $\dot{\gamma}$ is the shear rate. Carefully designed homogeneous flow experiments support this picture, revealing a decreasing $S$ and increasing $\phi$ upon reducing $\mu$, until the system jams $(S=0)$ when $\phi$ reaches a maximum and $\mu$ reaches a minimum value. [5]. As opposed to frictionless particles that jam isotropically at random close packing $\phi=\phi_{\mathrm{RCP}}$ and $\mu=0$, frictional particles jam with an anisotropic microstructure at $\phi=\phi_{J}\left(<\phi_{\mathrm{RCP}}\right)$ and $\mu=\mu_{J}>0$ [6].

Despite the conceptual power of this general result [4, its utility beyond homogeneous shear is limited. In pressure driven Poiseuille flow, for example, momentum conservation dictates that $\mu<\mu_{J}$ in a finite region around the centreline. In this region, the $[S(\mu), \phi(\mu)]$ rheology described above clearly predicts jamming with $S=0$ and $\phi=\phi_{J}$. This behaviour is not observed in experiments and particle-based simulations, however, which instead consistently show "sub-yielding" $(S>0)$ and sometimes "over-compaction" $\left(\phi>\phi_{J}\right)$, in regions where $\mu<\mu_{J}$ [7-11. Making quantitative predictions of practical flows that comprise contiguous regions of $\mu>\mu_{J}$ and $\mu<\mu_{J}$ thus requires more detailed constitutive models that capture both homogeneous rheology and the physics of subyielding and over-compaction that arise under inhomoge- neous conditions. Although these effects have been addressed separately in the literature, there are no models available that capture both effects simultaneously.

Sub-yielding and over-compaction under inhomogeneous flow occur in regions of vanishing shear rate, where the dynamics are completely governed by fluctuating particle motions [12 21]. These fluctuations propagate from flowing regions with $\mu>\mu_{J}$ into (nearly solid) regions with $\mu<\mu_{J}$, inducing particle rearrangements. This may allow the suspension to fluidise in otherwise solid regions, with $\phi_{J}<\phi<\phi_{\mathrm{RCP}}$.

Attempts at incorporating over-compaction in constitutive models are so far limited to linear extrapolation of the homogeneous $\phi(\mu)$ relation from regions with $\mu>\mu_{J}$ into regions with $\mu<\mu_{J}$ [20]. The shape of the resulting density profiles, however, qualitatively differs from experimental data [7, 8, 11. Sub-yielding, meanwhile, has been modelled by subjecting the fluidity (inverse viscosity) to a diffusion process [18, or by accounting for fluctuations in the expression for the suspension stress, with the fluctuation magnitude being computed using a transport equation borrowed from kinetic theory [9. Alternatively, a simpler account for fluctuations can be derived by spatially averaging, i.e. filtering, the stress over a volume that is small compared to the system size and large compared to the particle size [12, 22, 23]. This leads to an increase in the normal viscosity but leaves the shear viscosity unaffected, thereby reducing $\mu$ below $\mu_{J}$. Crucially, these sub-yielding models fail to account for microstructural changes due to fluctuations and therefore do not capture over-compaction.

In this Letter we address these shortcomings, providing an intuitive explanation of sub-yielding and overcompaction. We do so by incorporating shear rate fluctuations into a recent microstructure model $[24,28]$. When applied to inhomogeneous flows the resulting tensorial constitutive model predicts that fluctuations can: (i) isotropise the microstructure; (ii) increase $\phi$ above $\phi_{J}$; (iii) reduce $\mu$ below $\mu_{J}$. We compare the model predictions to those of particle-based simulations. 
Constitutive model.- The suspension stress tensor is modelled as 28]:

$$
\frac{\boldsymbol{\Sigma}}{\eta_{s}}=2\langle\boldsymbol{E}\rangle+\left[\frac{\alpha_{0}\langle\boldsymbol{E}\rangle}{\left(1-\frac{\phi}{\phi_{\mathrm{RCP}}}\right)^{2}}+\frac{\chi_{0}\left\langle\boldsymbol{E}_{c}\right\rangle}{\left(1-\frac{\xi}{\xi_{J}}\right)^{2}}\right]:\langle\boldsymbol{n n n n n}\rangle .
$$

Here $\boldsymbol{n}$ is the separation unit vector of interacting particle pairs, $\boldsymbol{L}=\boldsymbol{\nabla} \boldsymbol{u}^{T}$ is the velocity gradient tensor and $\boldsymbol{E}=\frac{1}{2}\left(\boldsymbol{L}+\boldsymbol{L}^{T}\right)$ is the rate of strain tensor, which we decompose into extensional $\boldsymbol{E}_{e}$ and compressive $\boldsymbol{E}_{c}$ parts:

$$
\boldsymbol{E}_{e}=\frac{1}{2} \boldsymbol{E}+\frac{1}{4}\|\boldsymbol{E}\| \boldsymbol{\delta}, \quad \boldsymbol{E}_{c}=\frac{1}{2} \boldsymbol{E}-\frac{1}{4}\|\boldsymbol{E}\| \boldsymbol{\delta} .
$$

Note that Eq. (2) is valid in 2D but not in 3D. Given the practical ubiquity of $2 \mathrm{D}$ shear we nonetheless proceed with Eq. (2).

In Eq. (1) the filter operator $\langle\cdot\rangle$ averages over particle pairs that are contained in a space-time, filtering volume which must be small compared to the spatial and temporal extents of the suspension and large compared to those of the fluctuations. When carrying out the filtering, it has been assumed in Eq. (1) [and in Eq. (5) below] that the fluctuations in the velocity gradient field are uncorrelated with the fluctuations in the pair separation vector, e.g. $\left\langle\boldsymbol{E}_{c} \boldsymbol{n n n n n}\right\rangle \approx\left\langle\boldsymbol{E}_{c}\right\rangle\langle\boldsymbol{n} \boldsymbol{n} \boldsymbol{n} \boldsymbol{n}\rangle$.

In Eq. (1) the jamming coordinate $\xi$ is defined as 28:

$$
\xi=-\frac{\langle\boldsymbol{n} \boldsymbol{n}\rangle:\left\langle\boldsymbol{E}_{c}\right\rangle}{\sqrt{\left\langle\boldsymbol{E}_{c}\right\rangle:\left\langle\boldsymbol{E}_{c}\right\rangle}},
$$

which acts as a proxy for the coordination number $Z$, i.e. the number of direct contacts per particle [27]. The first and second terms in Eq. (1) are, respectively, the stress induced by the fluid and by the particles. The latter contains lubrication and contact contributions, where $\alpha_{0}$ and $\chi_{0}$ are constants and $\xi_{J}$ is the value of $\xi$ at jamming.

In Eq. (1) the fourth-order moment $\langle\boldsymbol{n n n n n}\rangle$ of the orientation distribution function of $\boldsymbol{n}$ is expressed in terms of the second-order moment $\langle\boldsymbol{n n}\rangle$ using [29]:

$$
\begin{aligned}
& \left\langle n_{i} n_{j} n_{k} n_{l}\right\rangle=-\left\langle n_{m} n_{m}\right\rangle \times \\
& \frac{1}{(D+2)(D+4)}\left(\delta_{i j} \delta_{k l}+\delta_{i k} \delta_{j l}+\delta_{i l} \delta_{j k}\right) \\
& +\frac{1}{D+4}\left(\delta_{i j}\left\langle n_{k} n_{l}\right\rangle+\delta_{i k}\left\langle n_{j} n_{l}\right\rangle+\delta_{i l}\left\langle n_{j} n_{k}\right\rangle\right. \\
& \left.\quad+\left\langle n_{i} n_{j}\right\rangle \delta_{k l}+\left\langle n_{i} n_{k}\right\rangle \delta_{j l}+\left\langle n_{i} n_{l}\right\rangle \delta_{j k}\right) .
\end{aligned}
$$

The second-order moment $\langle\boldsymbol{n n}\rangle$ is related to the velocity gradient field with the following steady state balance equation [28]:

$$
\begin{gathered}
\mathbf{0}=\langle\boldsymbol{L}\rangle \cdot\langle\boldsymbol{n n}\rangle+\langle\boldsymbol{n n}\rangle \cdot\left\langle\boldsymbol{L}^{T}\right\rangle-2\langle\boldsymbol{L}\rangle:\langle\boldsymbol{n n n n n}\rangle \\
-\beta\left[\left\langle\boldsymbol{E}_{e}\right\rangle:\langle\boldsymbol{n n n n n}\rangle+\frac{\phi}{D(D+2)}\left(2\left\langle\boldsymbol{E}_{c}\right\rangle+\operatorname{Tr}\left(\left\langle\boldsymbol{E}_{c}\right\rangle\right) \boldsymbol{\delta}\right)\right] .
\end{gathered}
$$

The "pair association rate" $\beta$ controls the rate at which particle pairs are created and destroyed by fluid compression and extension, set respectively by $\boldsymbol{E}_{c}$ and $\boldsymbol{E}_{e}$. Eqs. (1.5) define a constitutive model for steady microstructure and stress in dense suspensions.

Incorporating fluctuations.- The shear rate consists of a mean $\dot{\gamma}=\|\langle\boldsymbol{E}\rangle\|=\sqrt{2\langle\boldsymbol{E}\rangle:\langle\boldsymbol{E}\rangle}$ and fluctuations. While in homogeneous flow, the fluctuations are subdominant to the mean, the fluctuations may dominate the mean in inhomogeneous flow, e.g. close to a Poiseuille centreline. In those regions, although the filtered $\boldsymbol{E}$ is (nearly) zero, the filtered $\boldsymbol{E}_{e}$ and $\boldsymbol{E}_{c}$ are non-zero, which is a consequence of the non-linearity of $\boldsymbol{E}_{e}$ and $\boldsymbol{E}_{c}$ in $\boldsymbol{E}$ [Eq. (2)]. Below we account for fluctuations in the model [Eqs. (115)] by filtering $\boldsymbol{E}_{e}$ and $\boldsymbol{E}_{c}$.

In order to express $\left\langle\boldsymbol{E}_{e}\right\rangle$ and $\left\langle\boldsymbol{E}_{c}\right\rangle$ in terms of $\langle\boldsymbol{E}\rangle$, we use that a fluctuating quantity $q=\langle q\rangle+q^{\prime}$ can be decomposed into its filtered $\langle q\rangle$ and its fluctuating $q^{\prime}$ components, and that $\left\langle q^{\prime}\right\rangle=0$. Filtering a non-linear function of $q$ gives additional terms. Specifically, filtering the absolute value of $q$ gives $\left\langle\left|\langle q\rangle+q^{\prime}\right|\right\rangle \approx|\langle q\rangle|+q_{\text {rms }}$ [30] where $q_{\mathrm{rms}}=\left\langle\left|q^{\prime}\right|\right\rangle$. Similarly, filtering Eq. (2) gives:

$$
\left\langle\boldsymbol{E}_{e}\right\rangle=\langle\boldsymbol{E}\rangle_{e}+\frac{1}{4} \dot{\gamma}_{\mathrm{rms}} \boldsymbol{\delta}, \quad\left\langle\boldsymbol{E}_{c}\right\rangle=\langle\boldsymbol{E}\rangle_{c}-\frac{1}{4} \dot{\gamma}_{\mathrm{rms}} \boldsymbol{\delta},
$$

where $\dot{\gamma}_{\mathrm{rms}}=\left\langle\left\|\boldsymbol{E}^{\prime}\right\|\right\rangle$ is the amplitude of the shear rate fluctuations.

In homogeneous shear flow, the fluctuating shear rate $\dot{\gamma}_{\text {rms }}$ vanishes when the mean shear rate $\dot{\gamma}$ vanishes. In inhomogeneous shear flows, on the other hand, $\dot{\gamma}_{\text {rms }}$ may remain finite when $\dot{\gamma} \rightarrow 0$, since fluctuations are propagating from nearby regions with finite $\dot{\gamma}$. In this limiting case, the dynamics are dominated by $\dot{\gamma}_{\text {rms }}$ and $\left\langle\boldsymbol{E}_{e}\right\rangle=-\left\langle\boldsymbol{E}_{c}\right\rangle=\frac{1}{4} \dot{\gamma}_{\mathrm{rms}} \boldsymbol{\delta}$. Inserting these expressions into the filtered microstructure and stress equations [Eqs. (1. 4. 5]] gives isotropic tensors for the microstructure $\langle\boldsymbol{n} \boldsymbol{n}\rangle \sim \boldsymbol{\delta}$ and the stress $\boldsymbol{\Sigma} \sim-\eta_{s} \dot{\gamma}_{\text {rms }} \boldsymbol{\delta}$, with negative normal stresses and zero shear stresses. This behaviour corresponds to a vanishing macroscopic friction coefficient $\mu$, below the jamming friction coefficient for homogeneous systems $\mu_{J}$. Our constitutive model similarly predicts isotropisation of the microstructure and stress in shear flow with superposed shear oscillations 28 .

Kolmogorov flow.- We apply the above model [Eqs. [1.6p] to steady 2D Kolmogorov flow, driven by a body force density $\boldsymbol{f}=\hat{f} \sin (2 \pi y / L) \boldsymbol{\delta}_{x}$ (with $\hat{f}$ the force amplitude) pointing in the $x$-direction and oscillating in the $y$-direction with a period $L$ (Fig. 1 1 ). We chose this flow to test our model, as it is possibly the simplest inhomogeneous shear flow without solid surfaces. In this inhomogeneous shear flow $\boldsymbol{L}=\partial_{y} u_{x} \boldsymbol{\delta}_{x} \boldsymbol{\delta}_{y}$ and the fluid mechanical profiles are periodic in $y$ and independent of $x$ and $t$. Figs. 1 $1 \mathrm{p}-\mathrm{c}$ show schematically the instantaneous and filtered profiles of the flow-gradient $x y$-component of the total deformation $\boldsymbol{E}$ and of its compressive part $\boldsymbol{E}_{c}$. Crucially, the filtered $E_{x y}=0$ on the centrelines 

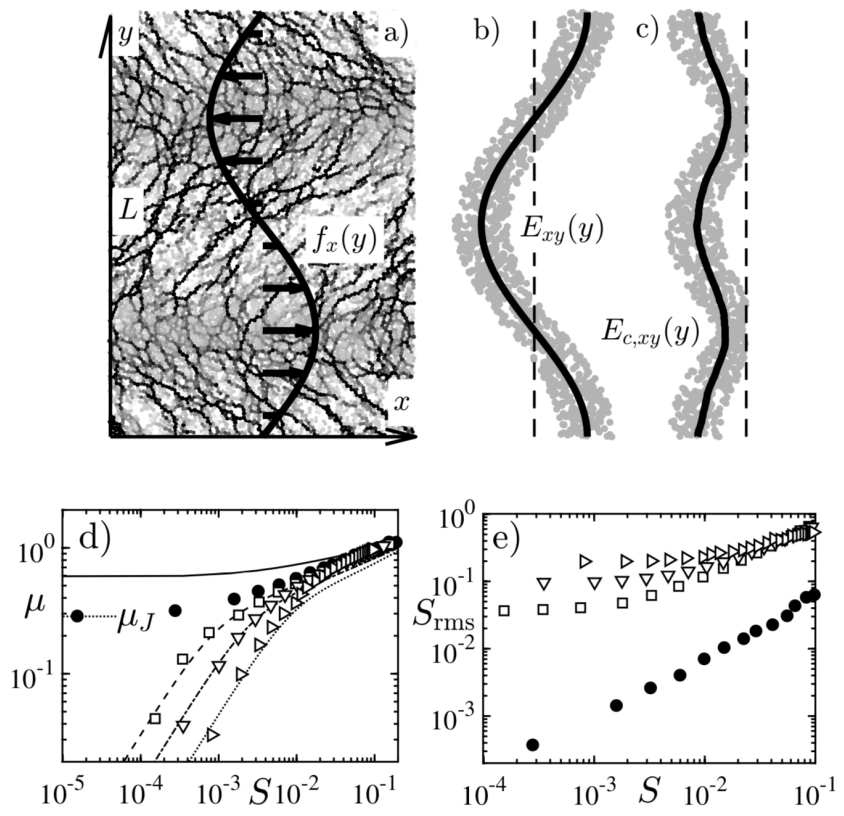

FIG. 1. (a) Snapshot of particle-based simulation of Kolmogorov flow with relative domain size $L / a=278$. The grey level indicates the particle pressure (black and white represent negative and positive, respectively) and the driving force $f_{x}(y)$ is sketched with the black line. (b, c) Sketches of the $x y$-component of $\boldsymbol{E}$ and $\boldsymbol{E}_{c}$ respectively, showing raw signals (grey) and filtered signals (black). The latter is zero at $y / L=0.25,0.75$ for $\langle\boldsymbol{E}\rangle$ but not for $\left\langle\boldsymbol{E}_{c}\right\rangle$. The dashed lines indicate the abscissas. (d) Macroscopic friction coefficient $\mu$ as a function of the non-dimensional shear rate $S$ predicted by simulation in homogeneous shear flow (filled circles) and in Kolmogorov flow for $L / a=278$ (open squares), 139 (open downward triangles) and 56 (open rightward triangles) and predicted by constitutive model for $S_{\mathrm{rms}}=0$ (solid line) $6 \times 10^{-3}$ (dashed line), $1.5 \times 10^{-2}$ (dash-dotted line) and $4.2 \times 10^{-2}$ (dotted line). (e) Non-dimensional shear rate fluctuations $S_{\mathrm{rms}}$ as a function of $S$ predicted by simulation. The markers are as in (d).

[at $y=L / 4(\bmod L / 2)]$, whereas the filtered $E_{c, x y}<0$. This difference arises due to the non-linearity of $\boldsymbol{E}_{c}$ in $\boldsymbol{E}$ mentioned above, and demonstrates that fluctuations produce normal stresses but no shear stresses, resulting in sub-yielding close to the centrelines.

Particle-based simulation.- We compare our constitutive model to particle-based simulations on 2D domains with dimensions in the $x$ and $y$-directions, respectively, of $L_{x}=200 a$ and $L=56 a, 139 a$ and 278a. We use $N \sim 10^{4}$ bidisperse frictional spheres (radii $a$ and 1.4a, stiffness $k$, density $\rho$ ) and a domain averaged volume fraction:

$$
\bar{\phi}=L^{-1} \int_{0}^{L} \phi(y) d y,
$$

of $\bar{\phi}=0.7$. The particles interact with each other through short-range lubrication and frictional contact forces [31] while drag forces between the particles and the suspending medium are omitted. Instead, the flow is driven by a $y$-dependent force in the $x$-direction $f_{0} \sin (2 \pi y / L) \boldsymbol{\delta}_{x}$ added to each particle. We set $f_{0} / k a=$ $10^{-8}$, sufficiently small for the particles to behave as hard, inertia-free spheres $\left(\rho \dot{\gamma} a^{2} / \eta_{s}<10^{-2}\right)$. The resulting driving force density is $\boldsymbol{f}=f_{0} n(y) \sin (2 \pi y / L) \boldsymbol{\delta}_{x}$ where $n(y)$ is the particle number density and the average force amplitude equals $\hat{f}=f_{0} \bar{n}=f_{0} N /\left(L L_{x}\right)$. Simulations are run until a statistically steady state is reached in the entire domain and profiles are computed thereafter over $\dot{\gamma} t \approx 20$, based on the maximum $\dot{\gamma}$ in the domain. We obtain velocity and structural profiles by averaging particle properties in $y$-bins, so that each single simulation provides a range of $\mu$ and $S$ values.

We also simulate 2D homogeneous shear flow, driven by Lees-Edwards boundary conditions, on a square domain with size $L=56 a$ and with $\phi=0.5-0.9$. By measuring the divergence of the stresses with increasing $\phi$, we found the jamming friction coefficient to be $\mu_{J}=0.285$ and the limiting volume fractions as $\phi_{J}=0.795$ and $\phi_{\mathrm{RCP}}=0.840$.

Model predictions of $\mu(S)$.- $^{-}$Fig. $1 \mathrm{~d}$ shows the simulation results on $(S, \mu)$-coordinates under homogeneous shear and in Kolmogorov flow for various $L / a$. The data points correspond to fixed $\phi$ values in homogeneous shear and to fixed $y$-coordinates in the Kolmogorov simulation. The inhomogeneous Kolmogorov flow simulation predicts sub-yielding, i.e. $S>0$ in regions where $\mu<\mu_{J}$ while the homogeneous shear simulation predicts the homogenous $\mu(S)$ rheology consistent with Ref. [5].

Shown in Fig. 1p are the simulated, non-dimensional shear rate fluctuations $S_{\mathrm{rms}}=\eta_{s} \dot{\gamma}_{\mathrm{rms}} / \Pi$ as a function of $S$ for the same cases as in Fig. 1 1 . The shear rate fluctuations $\dot{\gamma}_{\mathrm{rms}}=\left\langle\left|\partial_{y} u_{x}^{\prime}\right|\right\rangle$ are calculated based on instantaneous, local realisations of $\partial_{y} u_{x}$, computed by fitting a linear function to the spatial distribution of the instantaneous particle velocities in a box of size $6 a$. The data show an increase in $S_{\text {rms }}$ with a decrease in $L / a$ (that is, for steeper gradients of the driving force) and a (non)vanishing $S_{\mathrm{rms}}$ in the limit of $S \rightarrow 0$ for the (in)homogeneous shear flow.

Constitutive model predictions are plotted with lines in Fig. 11, with $\alpha_{0}=\chi_{0}=0.96, \xi_{J}=0.6$ and $\beta=4$. The latter two are not fitting parameters per se, but follow from $\phi_{\mathrm{RCP}}=0.840$ and $\phi_{J}=0.795$ 32. Each line is obtained by solving $\phi$ and $S$ from Eqs. (1 1 ) for various values of $\mu$ at fixed $S_{\text {rms }}$. $S_{\text {rms }}$ values are chosen to best match the simulation data in Fig. 1d (markers). They are somewhat smaller than $S_{\text {rms }}$ predicted by simulation (Fig. 1), reflecting that the constitutive model does not capture the correct quantitative relationship between $\mu$, $S$ and $S_{\text {rms. }}$. Nevertheless, the model predicts the correct qualitative behaviour, specifically $S_{\mathrm{rms}}>0$ results in sub-yielding, i.e. $\mu \rightarrow 0$ as $S \rightarrow 0$, with the effect being amplified as $S_{\text {rms }}$ is increased. 


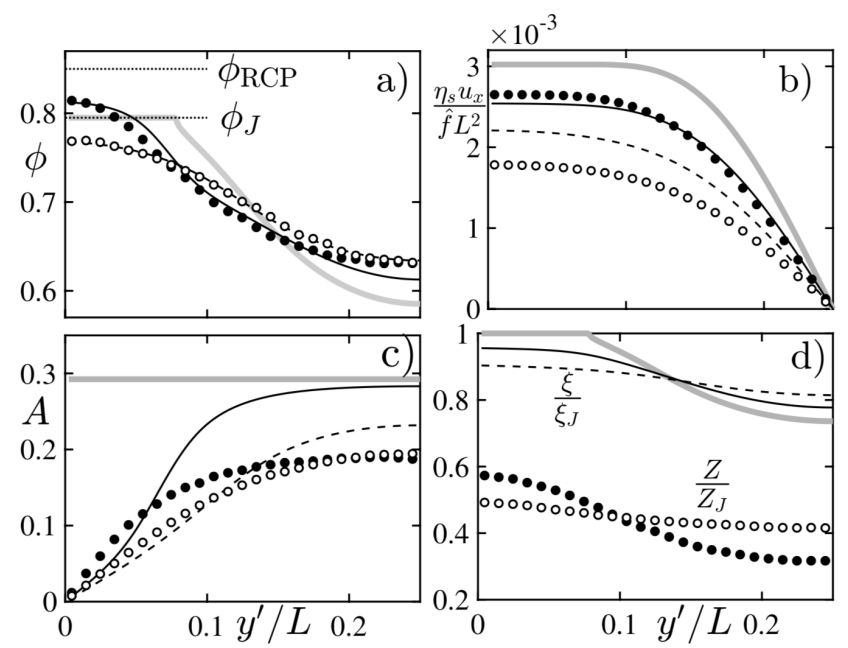

FIG. 2. Kolmogorov flow profiles as a function of the normalised distance to the nearest centreline $y^{\prime} / L$, predicted by constitutive model with $S_{\mathrm{rms}}=0$ (grey lines) $S_{\mathrm{rms}}=10^{-2}$ (solid black lines) and $S_{\mathrm{rms}}=10^{-1}$ (dashed black lines) and by simulations with $L / a=278$ (filled circles) and 56 (open circles). (a) Volume fraction $\phi$; (b) Normalised velocity $u_{x} /\left(\hat{f} L^{2} / \eta_{s}\right)$; (c) Anisotropy of particle contacts $A$; (d) Normalised coordination number $Z / Z_{J}$ in simulation and normalised jamming coordinate $\xi / \xi_{J}$ in constitutive model.

Model predictions of profiles.- Next we make predictions of the velocity and structural profiles in Kolmogorov flow by combining our constitutive model [Eqs. (1 6 ] ] with the (inertia-free) momentum balance $\boldsymbol{\nabla} \cdot \boldsymbol{\Sigma}+\boldsymbol{f}=\mathbf{0}$, whose $x$ and $y$-components reduce to:

$$
\Sigma_{x y}=\frac{\hat{f} L}{2 \pi} \cos (2 \pi y / L), \quad \Sigma_{y y}=- \text { constant. }
$$

We use three non-dimensional shear rate fluctuations (assumed constant throughout the domain) $S_{\mathrm{rms}}=0,10^{-2}$ and $10^{-1}$ where the former represents the homogeneous flow model and the latter two are chosen to match the model to the simulated $\phi$-profiles in Fig. 2 a (described below). These $S_{\text {rms }}$ values are different from those used in Fig. 1 1 which were chosen to match the simulated $\mu(S)$ profiles. These differences again indicate the quantitative discrepancies between model and simulation. We compute $\phi, \partial_{y} u_{x}$ and $\langle\boldsymbol{n n}\rangle$ in each $y$-coordinate for a given constant $\Sigma_{y y}$ from Eqs. (1, 6, 8) using NewtonRaphson and then iteratively updating $\Sigma_{y y}$ using the bisection method such that the integral volume fraction $\bar{\phi}$ [Eq. 77] approaches 0.7 .

Shown in Fig. 2 are profiles of the volume fraction $\phi$ (Fig. 2a), the non-dimensional suspension velocity $u_{x} \eta_{s} /\left(\hat{f} L^{2}\right)$ (Fig. $2 p$ ), the anisotropy $A$ of the particle contacts (Fig. 2 $\mathrm{c}$ ) and the coordination number $Z$ normalised by the value at homogeneous jamming $Z_{J}$ (Fig. $2 \mathrm{~d}) . Z$ is computed from the simulation output by counting contacting particle pairs (with $Z_{J}=3$ ), while $A$ is ob- tained by averaging $n_{x} n_{y}$ over all such pairs (with $\boldsymbol{n}$ the unit vector along the centre-to-centre line). In the constitutive model, $Z / Z_{J}$ and $A$ are represented, respectively, by $\xi / \xi_{J}$ and $-\left(\left\langle\boldsymbol{E}_{c}\right\rangle:\langle\boldsymbol{n n n n n}\rangle\right)_{x y} /\langle\boldsymbol{n n n}\rangle:\left\langle\boldsymbol{E}_{c}\right\rangle[28$. Due to symmetry Fig. 2 only shows the profiles over one quarter of the wavelength $L$.

Without fluctuations, i.e. following the homogeneous rheology, the constitutive model predicts a jammed region around the centrelines with $\dot{\gamma}=S=0$ and $\phi=\phi_{J}$ (grey lines in Fig. 2). Fluctuations induce two effects. The first is an increase of the repulsive normal stress relative to the imposed shear stress, which is evidenced by a decrease in $\mu$ for small $S$ in Fig. 11d. This increased normal stress drives particles away from the centrelines to the outer regions (Fig. 2a). In these outer regions the shear rate is larger and the particles generate more shear stress than in the centreline regions. This results in a lower non-dimensional velocity (Fig. 2p). The second effect is isotropisation (i.e. $A \rightarrow 0$ ) of the microstructure (Fig. 2k), resulting in fewer particle contacts at a given $\phi$ (Fig. 2d). This isotropisation allows $\phi$ to exceed $\phi_{J}$ and reduces the normal stress near the centrelines. These two competing effects may lead either to an increase in the volume fraction $\phi$ above $\phi_{J}$ (over-compaction, observed for $S_{\mathrm{rms}}=10^{-2}$ ) or to a reduction below $\phi_{J}$ (observed for $S_{\mathrm{rms}}=10^{-1}$ ) at the centrelines (Fig. 2 ).

Despite the qualitative agreement, there are quantitative differences between the constitutive model and the particle-based simulation. Fig. $2 \mathrm{~d}$ for instance shows that $\xi / \xi_{J}$ in the constitutive model is larger than $Z / Z_{J}$ in the simulation. There are many possible avenues for improving the quantitative accuracy of the model, e.g. by relaxing the assumption that velocity gradient fluctuations are uncorrelated with microstructure fluctuations or by using complex relationships between the material functions $\alpha_{0}, \chi_{0}$ and $\beta$ and the state variables $\phi,\langle\boldsymbol{L}\rangle$ and $\langle\boldsymbol{n n}\rangle$. However, having demonstrated that our model contains a (possibly minimal) set of physics that can simultaneously reproduce sub-yielding and overcompaction, we have chosen mathematical simplicity over quantitative accuracy, leaving the above developments as promising routes for further analysis.

Conclusion.- We have presented a tensorial model for the microstructure and stress in dense suspensions of frictional particles that includes the effect of fluctuations by applying a filtering to the microstructure balance equation. In doing so, we are able to predict sub-yielding and over-compaction, features common under practical flows but not predicted by homogeneous rheology models.

In addition to the potential model developments described above, further improvements to the predictive capacity for practical applications will require testing in complex geometries. We provide one such example in the Supplementary Material, namely a comparison between model and simulation predictions for pressure driven flow through a curved channel. Addressing the full details of 
this and other complex flows will be the next step towards a comprehensive fluid dynamical description of dense suspensions.

JJJG is supported by the Engineering and Physical Sciences Research Council of the United Kingdom Grant Number EP/N024915/1. CN acknowledges support from the Royal Academy of Engineering under the Research Fellowship scheme. We thank H. J. Wilson, J. D. Peterson and M. E. Cates for stimulating discussions.

\section{Supplementary Information: \\ Comparison between constitutive model and discrete element method for flow through a curved channel}

\section{Flow Problem}

We consider a two dimensional (2D) flow of a dense suspension in a curved channel. The channel has an inner radius of $R_{1}$ and an outer radius of $R_{2}$. The suspension flow is driven by a body force $\boldsymbol{f}=\hat{f} R_{1} r^{-1} \boldsymbol{\delta}_{\theta}$, with $\hat{f}$ a constant. The body force points in the azimuthal $\theta$ direction. The volume averaged $\cdots=2 \int_{R_{1}}^{R_{2}} \cdots r d r /\left(R_{2}^{2}-\right.$ $\left.R_{1}^{2}\right)$ body force equals $\bar{f}=2 \hat{f} R_{1}\left(R_{2}-R_{1}\right) /\left(R_{2}^{2}-R_{1}^{2}\right)$. No slip conditions are assumed on the walls. The flow is fully developed, i.e. the statistics of the flow only depend on the radial $r$-coordinate, but not on time $t$ nor on the $\theta$-coordinate.

\section{Constitutive Model}

The $\theta$-component of the momentum balance $\boldsymbol{\nabla} \cdot \boldsymbol{\Sigma}=$ $-\boldsymbol{f}$ reads: $\partial_{r} \Sigma_{r \theta}+2 r^{-1} \Sigma_{r \theta}=-\hat{f} R_{1} r^{-1}$, which, after integration, gives:

$$
\Sigma_{r \theta}=-\frac{\hat{f}}{2}+\frac{C_{1} R_{1}}{r^{2}},
$$

where $C_{1}$ is an unknown, to be determined, integration constant.

The $r$-component of the momentum balance reads: $\partial_{r} \Sigma_{r r}-r^{-1}\left(\Sigma_{\theta \theta}-\Sigma_{r r}\right)=0$. To simplify the analysis, we assume that $\left(\Sigma_{\theta \theta}-\Sigma_{r r}\right) / \Sigma_{r r}$ is equal to an unknown, to be determined constant:

$$
C_{2}=\overline{\left(\frac{\Sigma_{\theta \theta}-\Sigma_{r r}}{\Sigma_{r r}}\right)} .
$$

With this assumption, the $r$-momentum balance becomes: $\partial_{r} \Sigma_{r r}-r^{-1} \Sigma_{r r} C_{2}=0$, which, after integration, gives:

$$
\Sigma_{r r}=-C_{3}\left(\frac{r}{R_{1}}\right)^{C_{2}},
$$

where $C_{3}$ is yet another unknown, to be determined, integration constant.
The overall particle volume fraction in the system is denoted $\bar{\phi}$, i.e.:

$$
\bar{\phi}=\frac{2 \int_{R_{1}}^{R_{2}} \phi(r) r d r}{R_{2}^{2}-R_{1}^{2}} .
$$

The velocity profile $U_{\phi}$ is related to the deformation rate $E_{r \phi}=\left(\partial_{r}-r^{-1}\right) U_{\phi}$ through $U_{\phi}(r)=$ $U_{\phi}\left(R_{1}\right)+r \int_{R_{1}}^{r} d r^{\prime} E_{r \phi}\left(r^{\prime}\right) / r^{\prime}$. Demanding that $U_{\phi}\left(R_{1}\right)=$ $U_{\phi}\left(R_{2}\right)=0$ gives:

$$
0=r \int_{R_{1}}^{R_{2}} \frac{E_{r \phi}\left(r^{\prime}\right)}{r^{\prime}} d r^{\prime} .
$$

Provided $C_{1}, C_{2}$ and $C_{3}$, we use Newton-Raphson (in an inner loop) to find $\phi, E_{r \phi}$ and $\langle\boldsymbol{n n}\rangle$ in each $r$ coordinate that satisfy Eqs. (9, 11) and Eqs. (1-6) in the main text. We use an outer loop to update $C_{2}$ using Eq. (10) and we use Newton Raphson to update $C_{1}$ and $C_{2}$ in order to satisfy Eqs. 12, 13.

We use the constitutive model to compute three flow cases with different radii of curvature. The corresponding radius ratios are $R_{2} / R_{1}=1,1.5$ and 2 , respectively. In order to have a good match between the velocity magnitude predicted by the constitutive model and the discrete element method (DEM) (described below) we use for these three cases $\alpha_{0}=\chi_{0}=0.96,1.15$ and 1.26, respectively. The other parameters in the constitutive model are $\phi_{\mathrm{RCP}}=0.85, \phi_{J}=0.795, \bar{\phi}=0.7$ and $S_{\mathrm{rms}}=E_{r \phi, \mathrm{rms}} \eta_{s} / C_{3}=10^{-2}$.
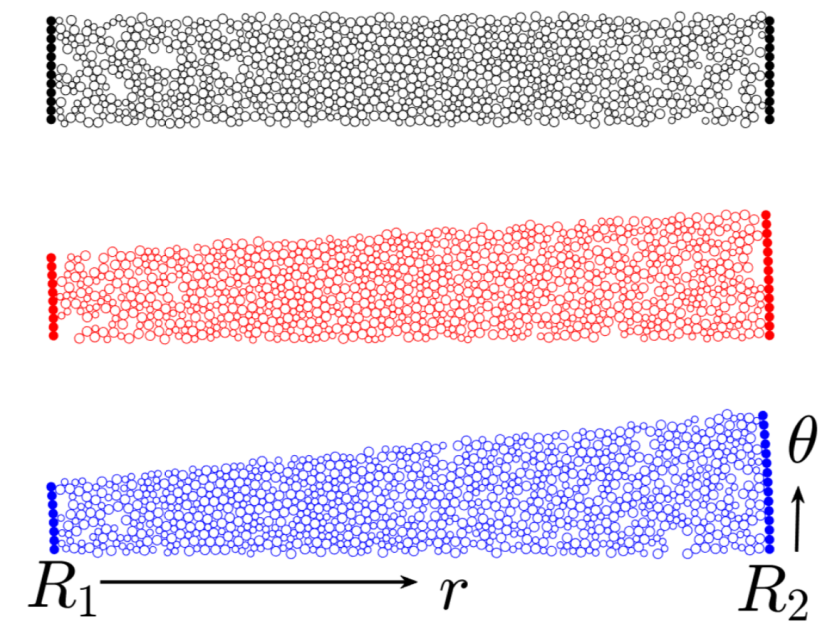

FIG. 3. Particles in the DEM simulations with $R_{2} / R_{1}=1$ (top), 1.5 (middle) and 2 (bottom). The empty particles are free and the filled particles are stationary and represent the solid boundaries.

\section{Discrete Element method}

We compare the results from the constitutive model to those of a DEM. For this purpose we use an in-house 
DEM code, that is similar to the one that is described in the main text. In this code, solid walls are implemented as a collection of fixed particles and boundary conditions are implemented in the azimuthal direction that are periodic over an adjustable angle $\Delta \theta$.

The flow in the DEM is driven by applying a force to each particle. This force points in the $\theta$-direction and it depends on the $r$-direction as $f_{0}\left(R_{1} / r\right) \boldsymbol{\delta}_{\theta}$. Here $f_{0}$ is a constant. Assuming a homogeneous number density of particles $n=2 N /\left[\Delta \theta\left(R_{2}^{2}-R_{1}^{2}\right)\right]$ this force distribution corresponds to a volume averaged body force density of $\bar{f}=2 n f_{0} R_{1}\left(R_{2}-R_{1}\right) /\left(R_{2}^{2}-R_{1}^{2}\right)$.

We use $N=1000$ bi-disperse spheres with radii $a$ and $1.4 a$ and a radial domain size of $\Delta R / a=214$, with $\Delta R=$ $R_{2}-R_{1}$. We simulate three cases with $R_{2} / R_{1}=1,1.5$ and 2 which correspond to a domain angle of $\Delta \theta=0,0.06$ and 0.1 and to a domain aspect ratio of $\Delta \theta R_{1} / \Delta R=$ 6.7, 8.3 and 10, respectively. The three computational domains with the simulated particles are illustrated in Fig. 3 .

\section{Comparison}

Fig. 4 compares the constitutive model to the DEM in terms of the profiles of the volume fraction $\phi$, the nondimensional suspension velocity $U_{\phi} \eta_{s} /\left(\bar{f} \Delta R^{2}\right)$, the contact microstructure anisotropy $A$ and the scaled coordination number, denoted $Z / Z_{J}$ in DEM and $\xi / \xi_{J}$ in the constitutive model. The definitions of these quantities are given in the main text. Similar as in the Kolmogorov flow (described in the main text) the effects of flow inhomogeneity are concentrated in the region of vanishing shear stress. When the aspect ratio $R_{2} / R_{1}$ increases, this region moves from the channel center towards the inner wall. This region is characterised by an isotropisation, i.e. $A \rightarrow 0$ (Fig. 4 4 ) and an over-compaction of the microstructure, i.e. $\phi>\phi_{J}$ (Fig. 4a). As $R_{2} / R_{1}$ increases, the model also correctly captures an increase near the walls of the particle density (Fig. 4 a) and the coordination number (Fig. 4a). This redistribution results in a decrease in the suspension velocity (Fig. 4b).

* jurriaangillissen@gmail.com

[1] Jonathan J Stickel and Robert L Powell, "Fluid mechanics and rheology of dense suspensions," Annu. Rev. Fluid Mech. 37, 129-149 (2005).

[2] Aaron S Baumgarten and Ken Kamrin, "A general constitutive model for dense, fine-particle suspensions validated in many geometries," Proceedings of the National Academy of Sciences 116, 20828-20836 (2019).

[3] Morton M Denn and Jeffrey F Morris, "Rheology of nonbrownian suspensions," Annual review of chemical and biomolecular engineering 5, 203-228 (2014).
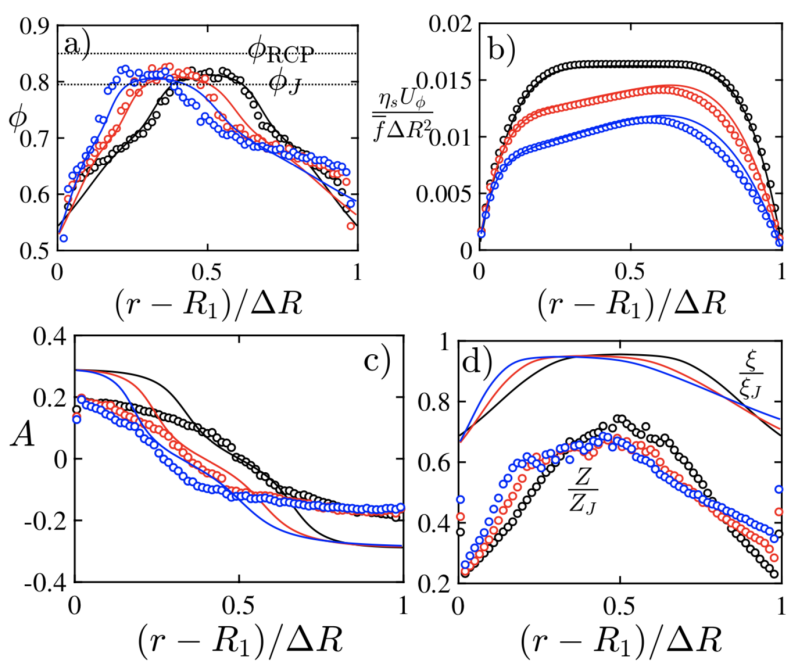

FIG. 4. Comparison between the constitutive model (lines) and the DEM (markers) in terms of the profiles of the volume fraction (a), the suspension velocity (b), the contact microstructure anisotropy (c) and the coordination number (d) for $R_{2} / R_{1}=1$ (black), 1.5 (red) and 2 (blue).

[4] Élisabeth Guazzelli and Olivier Pouliquen, "Rheology of dense granular suspensions," J. Fluid Mech. 852 (2018).

[5] François Boyer, Élisabeth Guazzelli, and Olivier Pouliquen, "Unifying suspension and granular rheology," Physical Review Letters 107, 188301 (2011).

[6] Matthieu Wyart and M. E. Cates, "Discontinuous shear thickening without inertia in dense non-brownian suspensions," Phys. Rev. Lett. 112, 098302 (2014).

[7] RE Hampton, AA Mammoli, AL Graham, N Tetlow, and SA Altobelli, "Migration of particles undergoing pressure-driven flow in a circular conduit," Journal of Rheology 41, 621-640 (1997).

[8] MK Lyon and LG Leal, "An experimental study of the motion of concentrated suspensions in two-dimensional channel flow. part 1. monodisperse systems," Journal of Fluid Mechanics 363, 25-56 (1998).

[9] Prabhu R Nott and John F Brady, "Pressure-driven flow of suspensions: simulation and theory," Journal of Fluid Mechanics 275, 157-199 (1994).

[10] Kyongmin Yeo and Martin R Maxey, "Numerical simulations of concentrated suspensions of monodisperse particles in a poiseuille flow," Journal of fluid mechanics $\mathbf{6 8 2}$, 491-518 (2011).

[11] Sangwon Oh, Yi-qiao Song, Dmitry I Garagash, Brice Lecampion, and Jean Desroches, "Pressure-driven suspension flow near jamming," Physical review letters 114, 088301 (2015).

[12] Ryan M Miller and Jeffrey F Morris, "Normal stressdriven migration and axial development in pressuredriven flow of concentrated suspensions," Journal of nonnewtonian fluid mechanics 135, 149-165 (2006).

[13] Lucio Isa, Rut Besseling, and Wilson CK Poon, "Shear zones and wall slip in the capillary flow of concentrated colloidal suspensions," Physical Review Letters 98, 198305 (2007). 
[14] Ken Kamrin and Martin Z Bazant, "Stochastic flow rule for granular materials," Physical Review E 75, 041301 (2007).

[15] Julie Goyon, Annie Colin, G Ovarlez, A Ajdari, and L Bocquet, "Spatial cooperativity in soft glassy flows," Nature 454, 84-87 (2008).

[16] Lydéric Bocquet, Annie Colin, and Armand Ajdari, "Kinetic theory of plastic flow in soft glassy materials," Physical review letters 103, 036001 (2009).

[17] Olivier Pouliquen and Yoel Forterre, "A non-local rheology for dense granular flows," Philosophical Transactions of the Royal Society A: Mathematical, Physical and Engineering Sciences 367, 5091-5107 (2009).

[18] Ken Kamrin and Georg Koval, "Nonlocal constitutive relation for steady granular flow," Physical Review Letters 108, 178301 (2012).

[19] Mehdi Bouzid, Martin Trulsson, Philippe Claudin, Eric Clément, and Bruno Andreotti, "Nonlocal rheology of granular flows across yield conditions," Physical review letters 111, 238301 (2013).

[20] Brice Lecampion and Dmitry I Garagash, "Confined flow of suspensions modelled by a frictional rheology," Journal of Fluid Mechanics 759, 197-235 (2014).

[21] Thomas Pähtz, Orencio Durán, David N De Klerk, Indresan Govender, and Martin Trulsson, "Local rheology relation with variable yield stress ratio across dry, wet, dense, and dilute granular flows," Physical review letters 123, 048001 (2019).

[22] P Mills and P Snabre, "Rheology and structure of concentrated suspensions of hard spheres. shear induced particle migration," Journal de Physique II 5, 1597-1608 (1995).

[23] Jeffrey F Morris and Fabienne Boulay, "Curvilinear flows of noncolloidal suspensions: The role of normal stresses," Journal of rheology 43, 1213-1237 (1999).

[24] JJJ Gillissen and HJ Wilson, "Modeling sphere suspension microstructure and stress," Physical Review E 98, 033119 (2018).

[25] JJJ Gillissen and HJ Wilson, "Effect of normal contact forces on the stress in shear rate invariant particle suspensions," Physical Review Fluids 4, 013301 (2019).
[26] JJJ Gillissen and HJ Wilson, "Taylor-couette instability in sphere suspensions," Physical Review Fluids 4, 043301 (2019).

[27] Jurriaan JJ Gillissen, Christopher Ness, Joseph D Peterson, Helen J Wilson, and Michael E Cates, "Constitutive model for time-dependent flows of shear-thickening suspensions," Physical Review Letters 123, 214504 (2019).

[28] JJJ Gillissen, C Ness, JD Peterson, HJ Wilson, and ME Cates, "Constitutive model for shear-thickening suspensions: Predictions for steady shear with superposed transverse oscillations," Journal of Rheology 64, 353-365 (2020).

[29] E J Hinch and L G Leal, "Constitutive equations in suspension mechanics. Part 2. Approximate forms for a suspension of rigid particles affected by Brownian rotations," J. Fluid Mech. 76, 187-208 (1976).

[30] When $|\langle q\rangle| \gg q_{\text {rms }}$ we have $\left\langle\left|\langle q\rangle+q^{\prime}\right|\right\rangle \approx|\langle q\rangle| \approx|\langle q\rangle|+$ $q_{\mathrm{rms}}$ and when $|\langle q\rangle| \ll q_{\mathrm{rms}}$ we have $\left\langle\left|\langle q\rangle+q^{\prime}\right|\right\rangle \approx q_{\mathrm{rms}} \approx$ $|\langle q\rangle|+q_{\mathrm{rms}}$. For the intermediate case $|\langle q\rangle| \sim q_{\mathrm{rms}}$ we interpolate between these limiting cases, giving: \langle|$\langle q\rangle+$ $q^{\prime}|\rangle \approx|\langle q\rangle|+q_{\mathrm{rms}}$.

[31] Oliver Cheal and Christopher Ness, "Rheology of dense granular suspensions under extensional flow," J. Rheol. 62, 501-512 (2018).

[32] To determine $\beta$ we use that, in the isotropic (microstructure and stress) limit where $\dot{\gamma}_{\text {rms }} \gg \dot{\gamma}$, we have $\xi=\phi / \sqrt{2}$ [28], and demanding jamming at $\phi_{\mathrm{RCP}}$ gives: $\xi_{J}=\phi_{\mathrm{RCP}} / \sqrt{2}$. We furthermore use that, in the simple-shear limit, where $\dot{\gamma}_{\text {rms }} \ll$ $\dot{\gamma}$, we have $\xi=\phi\left(5 \beta^{2}-4 \beta+32\right) /\left(2 \beta^{2}+8 \beta+64\right)$ [28, and demanding jamming at $\phi_{J}$ gives: $\xi_{J}=$ $\phi_{J}\left(5 \beta^{2}-4 \beta+32\right) /\left(2 \beta^{2}+8 \beta+64\right)$. Combining these two expressions for $\xi_{J}$ gives:

$\beta=\frac{\sqrt{200 \sqrt{2} \phi_{\mathrm{RCP}} \phi_{J}-56 \phi_{\mathrm{RCP}}^{2}-156 \phi_{J}^{2}}-2 \sqrt{2} \phi_{\mathrm{RCP}}-2 \phi_{J}}{\sqrt{2} \phi_{\mathrm{RCP}}-5 \phi_{J}}$,

which is around four for $\phi_{\mathrm{RCP}}=0.84$ and $\phi_{J}=0.795$. 